\title{
WIND ENERGY ROTOR SPEED CONTROL
}

\author{
David Westine, Greg Hilker, Fred Jenkins \\ Montana Tech / Ball Aerospace / ASiMI
}

\begin{abstract}
A grant involving the Department of Energy, the Montana Science and Technology Alliance, the Montana University System, and industry support from the Montana Power Corporation has funded construction of a wind energy test bed at the Rice Ridge Renewable Energy Park. Two small wind machines were planned for the initial phase of the Rice Ridge site shown in Figure 1. Located near Norris, MT which is approximately half way between Butte (Montana Tech) and Bozeman (Montana State University), the Park is designed to rapidly fatigue test fiberglass rotor blades and to procure an accurate strain history until fatigue failure. The first wind machine installed was a Bergey Excel. It has a 23 foot diameter fiberglass three bladed upwind rotor that drives a 10 kilowatt permanent magnet alternator and is mounted on a 60 foot tilt-down lattice tower. The second wind machine uses a Zephyr 22 kilowatt permanent magnet alternator driven by the same type of rotor used on the Bergey machine and is mounted on a 60 foot tilt-down tubular tower.
\end{abstract}

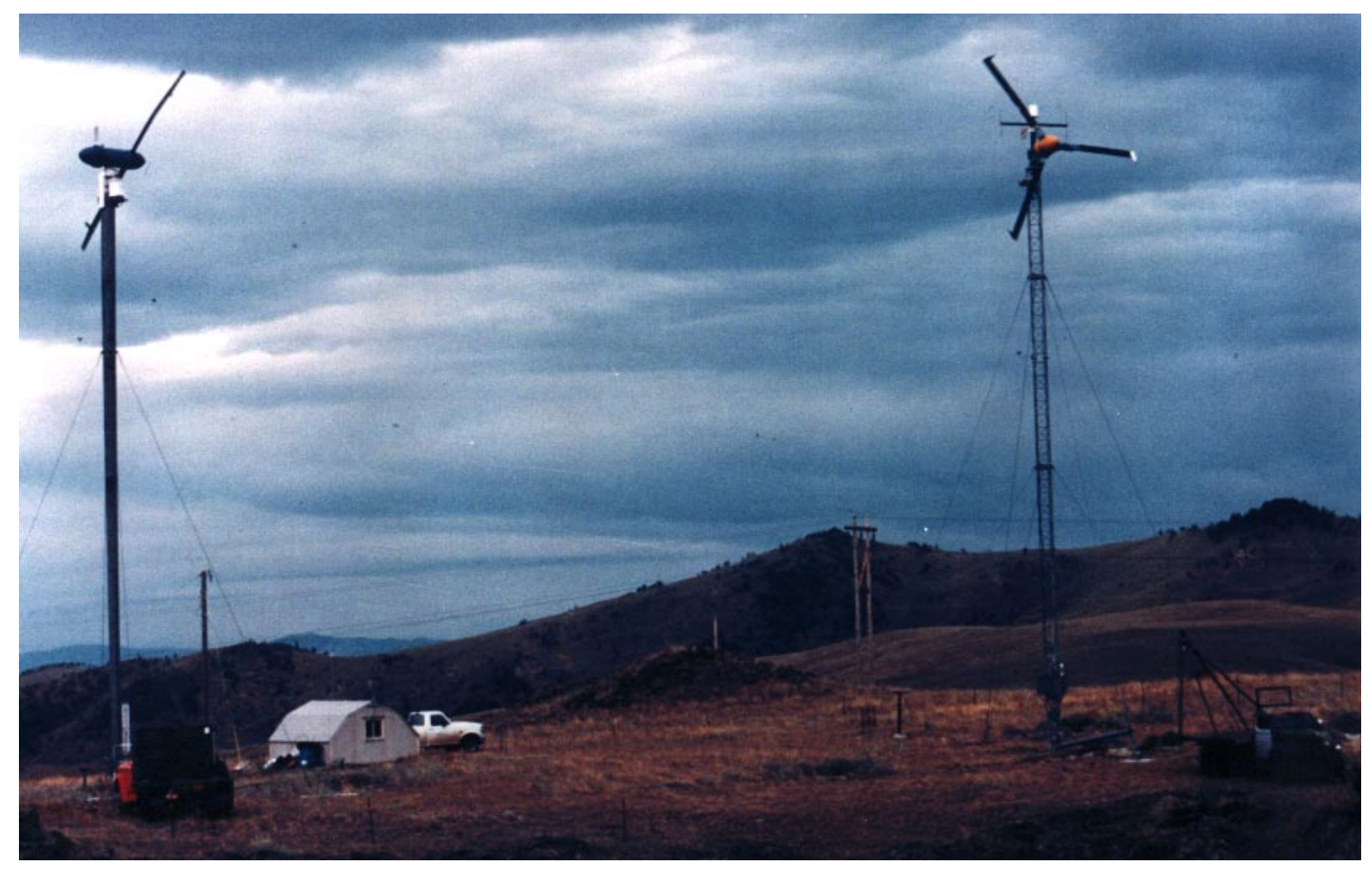

Figure 1 Rice Ridge Renewable Energy Park 
The Bergey has been modified to accommodate two different control systems. Both controllers are designed to limit rotor speed for experiments to test the fiberglass coupons. The rotor blades have also been equipped with a nine channel data acquisition telemetry system to procure strain data from each of the three blades (see Figure 2.) A fiberglass coupon, instrumented with strain gauges and designed for maximum strain, is inserted between the hub and the rotor blade. A capture device limits the maximum strain and secures the rotor blade when the coupon fails. This system can rapidly test fiberglass blade sections to fatigue failure without the expense of constructing a complete blade.

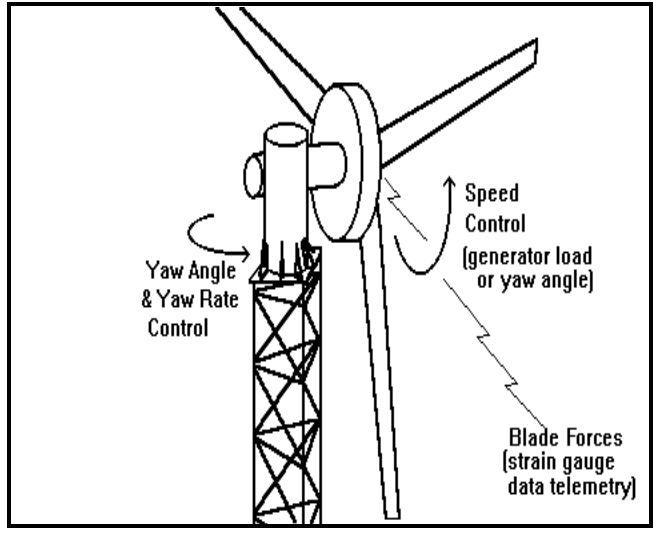

Figure 2 Control and Telemetry
One of the speed control systems is a closed-loop proportional-integral-derivative (PID) ${ }^{1}$ system that loads the alternator with a resistive heater bank. System hardware is composed of an Omega CN8500 series PID temperature controller, a Chromalox 4117 silicon controlled rectifier (SCR) ${ }^{2}$ AC power controller, and an Ohio Semitronics frequency transducer that monitors the frequency (rotor speed) of the alternator (see Figure 3.) The frequency transducer outputs a 4 to $20 \mathrm{~mA}$ current corresponding to a rotor speed of 0 to 380 revolutions per minute (RPM), respectively. Rotor speed is the process variable ${ }^{2}$ and is input to the PID controller. Maximum rotor RPM is the setpoint ${ }^{2}$ entered into the

PID controller by the operator. The rotor will free-spin up to the setpoint, and then the PID control will be implemented after the setpoint is exceeded. The PID controller causes the AC power controller to fire and load the alternator.

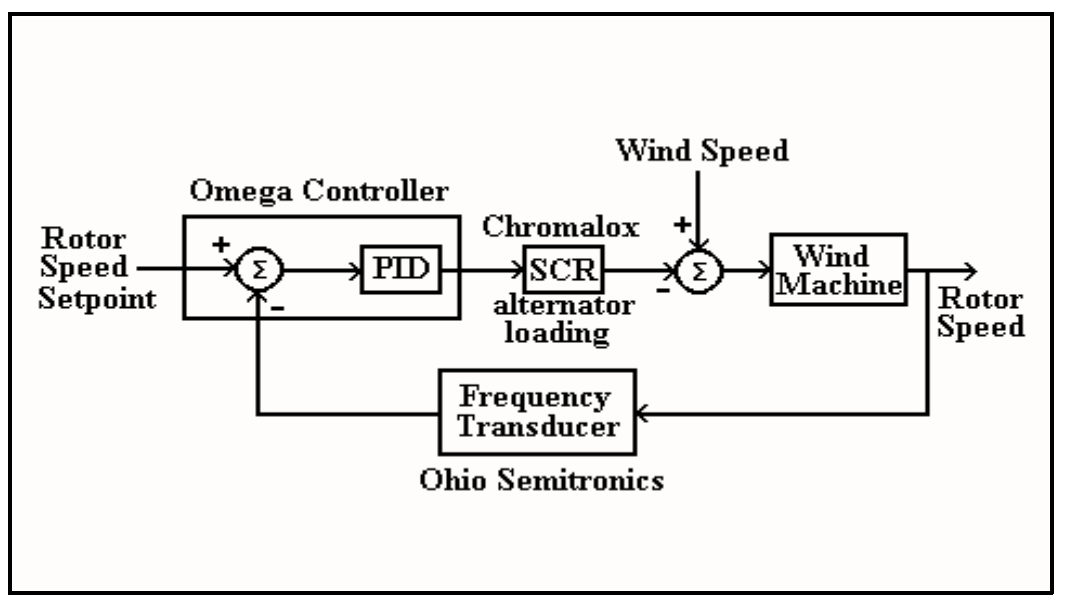

Figure 3 Wind Turbine Controller - Alternator Loading 
The Omega PID controller was originally designed for temperature control either through heating and/or through cooling. In order to control the rotor speed of the wind machine, the controller is set in the cooling mode and outputs a $4 \mathrm{~mA}$ current when the rotor speed is below the setpoint (the temperature is cooler than thermostat setpoint). When the rotor speed exceeds the setpoint (temperature is above the thermostat setpoint), the PID operation outputs a current that depends on the rotor speed error, on its time integral, and on its time derivative (PID). This output current is input to the SCR power controller which uses a pulse-width-modulation scheme to control the switching (duty cycle) of resistive heaters to the alternator. As the control current increases, the duty cycle increases, thereby causing the apparent rotor torque to increase. This increase lowers the rotor speed until the integrated error between rotor speed and rotor setpoint decreases and an error of zero is reached. The Omega PID controller is a self-tuning controller, and that option was used on the wind machine with satisfactory results. As shown in Figure 3, wind speed is the disturbance into the system and is continuously driving the rotor faster or slower than the setpoint. Rotor speed control about the setpoint of 40 RPM is shown in Figure 4.

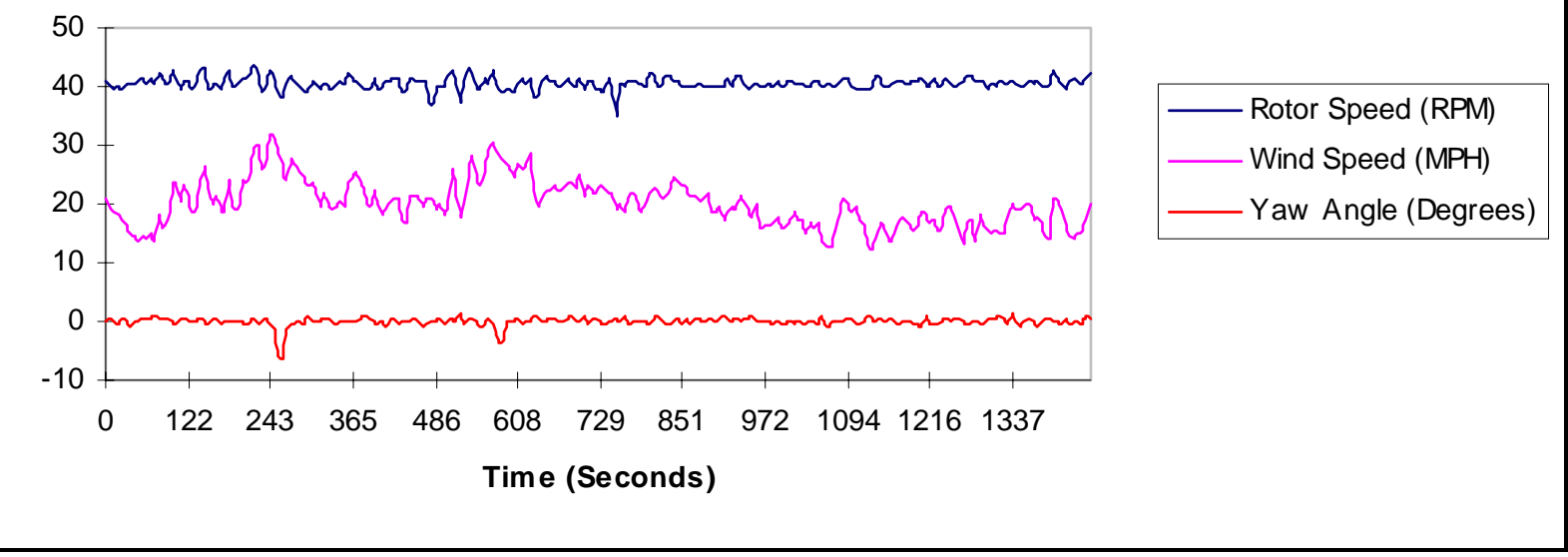

Figure 4 Rotor Speed Control - Alternator Loading

The other speed control system is a microprocessor embedded controller that turns the machine out of the wind in overspeed conditions by means of a yaw motor-drive system. Yaw, in this case, is defined as the angle between the wind and the rotor axis-of-rotation where a zero degree yaw angle is when the rotor is aligned into the wind and a $90^{\circ}$ yaw angle is when the rotor is turned at a right angle to the wind direction. The yaw motor interfaces with a variable frequency drive that is controlled by the microprocessor. There are three inputs to the microprocessor: yaw position, rotor speed (RPM), and wind speed. Yaw position is measured by means of a small free-turning tail vane mounted on the wind machine and connected to a rotary potentiometer. Through the use of software, different control schemes can be implemented to control the rotor speed via yaw angle. Figure 5 illustrates the rotor speed control system using yaw. 


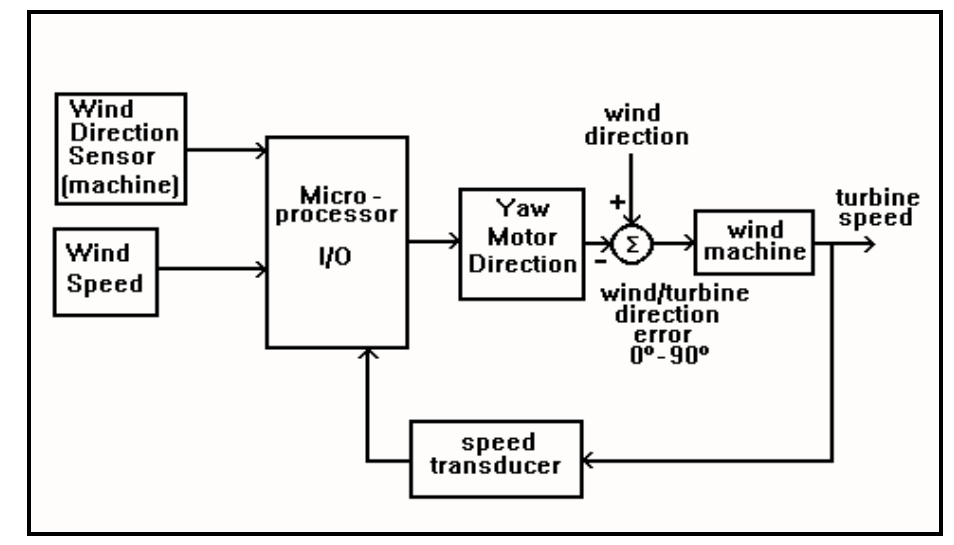

Figure 5 Wind Turbine Controller - Yaw Angle

The three rotor blade coupons have been instrumented with strain gauges to provide flap, edge and axial (or torsion) strain. This results in nine channels of data be telemetered from the spinning rotor. The maximum rotor speed is assumed to be 300 RPM, and at least the first six harmonics of the maximum fundamental frequency are to be acquired. Nine analog to digital converters (ADC) are used to simultaneously sample all nine channels, and then a universal asynchronous receiver/transmitter (UART) is used to serially send the data to a spread spectrum wireless modem. A tenth null channel is used for synchronizing the data train. Anti-aliasing filter, strain gauge conditioning, and gain amplifier circuits precede the ADCs.

Data are then collected on the ground in a personal computer and either displayed or stored on disk. Sample rates of 440 Hertz per channel for three channels (one blade) or 175 Hertz per channel for nine channels (all three blades) are achieved. Power to the system is supplied through a DC/DC converter and battery that is charged by commutation on the rotor. Total cost for the telemetry system design and construction is about $\$ 5,000$. Approximate production costs including strain modules would be $\$ 3,850$. Currently, the system collects strain data for the grant research and for graduate student research. Other similar systems sample until a buffer is filled and then stop sampling while the buffer is telemetered to the ground. These systems cost about $\$ 20,000$.

The computers and tools used to work on the wind machines are housed in a 12 by 16 foot instrument shack which provides work space and a programming/data analysis area for the researchers. Utility power provides heat and light for the shack. The Rice Ridge Energy Park is located so that radio telemetry can provide a communications link both to Butte and to Bozeman. Montana Tech's radio repeater stations are used to transmit and receive data between the college and the instrument shack.

Once the fiberglass coupon has been designed, instrumented, and installed, the rotor will have a maximum angular speed that ensures a full strain cycle per revolution. With the alternator load properly applied by the PID feedback control system, the rotor speed will be maintained at or below 
the maximum RPM in winds less than that value where full power is produced by the alternator. In these winds, the yaw control system orients the rotor into the wind direction in order to maintain the rotor speed at or near its maximum RPM value. If the wind speed exceeds that value which produces the maximum rated power output of the alternator, the rotor will then start to exceed the setpoint RPM even though the alternator is producing its maximum counter-torque. When this occurs, the yaw control system will start to turn the rotor out of the wind, lowering the amount of wind power driving the rotor. As a result, the rotor speed will be reduced. Since the wind speed and direction are continuously changing, the ability to control rotor speed using yaw angle is difficult. Finally, when wind speed exceeds a maximum value where it is no longer safe to try to maintain maximum RPM, the yaw control system should then keep the rotor turned $90^{\circ}$ out of the wind to minimize the windrotor interactions. Also, when wind speeds are too low to turn the rotor, the yaw motor should be off so light variable winds don't unnecessarily cause the yaw control system to search for the proper wind direction. In summary, rotor speed control strategy is as follows:

1. The wind is tracked by using the yaw control system, and the alternator is properly loaded to maintain maximum rotor speed until the wind speed exceeds the value that causes maximum rated power of the alternator.

2. When maximum rated power of the alternator is exceeded, the yaw control system starts to turn the machine out of the wind to maintain maximum rotor speed.

3. When the wind speed exceeds an absolute maximum average speed, the wind machine is turned $90^{\circ}$ out of the wind to protect the fiberglass coupon.

As a result of this control strategy, the yaw control system works in four modes:

1. Yaw motor off when wind speeds are below cut-in.

2. Tracking mode when power in the wind is less than alternator rated power.

3. Overspeed mode when the wind power exceeds alternator rated power.

4. Cut-out mode when wind speed exceeds strain ratings of the fiberglass coupon.

The controller used for yaw orientation is the Intel 8052 microcontroller. This is a Basic language, eight bit, floating point controller. Students and staff at Montana Tech have developed a printed circuit board called REmote Data ACquisistion System (REDACS) that uses the 8052 and associated RAM, ROM, and a four channel multiplexed 12 bit ADC and digital to analog conversion (DAC) circuitry. The REDACS then provides analog input-output (I/O) and digital I/O. The inputs to REDACS are:

1. Rotor speed

2. Wind speed

3. Yaw angle

4. Angle of machine to tower (wind machine 2).

The outputs from REDACS for yaw control are:

1. Analog output for yaw motor speed

2. Digital output for yaw motor on/off

3. Digital output for yaw motor direction.

Since the yaw control system operates under four separate modes, an intelligent controller must be implemented using the REDACS. 
The research/thesis ${ }^{3}$ portion of Greg Hilker's master's degree at Montana Tech investigated the yaw control system of the wind machine. To test different yaw control strategies for rotor speed control, Hilker unloaded the alternator to produce a rotor response that was the most sensitive to wind speed changes, and that would be the most difficult to control. The rotor response was first modeled in Matlab using Simulink. Wind, yaw, and rotor speed data were recorded from the wind machine with no load on the alternator. These data were recorded in 20 to 30 mile per hour winds with the yaw angle set at $10^{\circ}$ intervals from $0^{\circ}$ (aligned with the wind) to $90^{\circ}$ (turned out of the wind). System identification ${ }^{4}$ techniques, taught in a graduate controls course at Montana Tech, were implemented by using Matlab to approximate a linear model of the rotor response. The minimization of the mean-squared-error between the data and the model ${ }^{4}$ was used to produce models from a zero order to a tenth order. The mean-squared-error was then used as a measure of the best model approximation. A second order model produced the best fit. A nonlinear model was also simulated using Simulink, and it proved to have a slightly lower meansquared-error than the linear model. These two models were then tested at the different yaw angles where the wind was scaled by the cosine of the yaw angle prior to input. The nonlinear model consistently had a lower mean-squared-error than the linear model in all of the off-center yaw angles. Therefore, the nonlinear model was used to test the controller strategies.

First, a simple controller was tested on the model. It involved a constant yaw rate of 1.2 RPM and would turn the rotor out of the wind when the maximum rotor RPM was exceeded. Then turn it back into the wind when the rotor speed decreased below the maximum RPM setpoint. This fixed yaw-rate controller is an event-driven strategy ${ }^{2}$ where the wind machine is turned out of the wind in overspeed until the rotor is no longer in overspeed and then returns to the tracking mode.

The second controller was also event-driven, but used both rotor speed and rotor acceleration to determine the status of the yaw motor. This method also used a constant yaw rate of 1.2 RPM. When the rotor speed exceeds the maximum RPM setpoint and the rotor acceleration is positive, the yaw motor is activated and starts turning the machine out of the wind. The yaw motor continues to turn the rotor out of the wind until the rotor acceleration is negative, and at this time the yaw controller returns to the tracking mode but maintains yaw angle at the present position. This process continues until either the rotor speed decreases below the maximum RPM setpoint or until the acceleration returns to a positive value. If rotor speed decreases below the setpoint, the yaw controller continues in tracking mode, but maintains the yaw angle at zero degrees. If rotor acceleration returns to a positive value, the yaw controller will turn the wind machine further out of the wind.

Finally, the third controller used a proportional derivative (PD) $)^{1,2,4}$ type of control strategy where the rotor speed error and the derivative of the rotor speed error (rotor acceleration) were separately weighted and summed. This control signal then determines the yaw motor rate between 0.6 RPM and 3.0 RPM. The controller yaws the wind machine either out of the wind or into the wind at a variable rate that depends on the PD controller output. Since both the actual rotor speed error and the rotor acceleration are used to drive the yaw motor, the PD is a predictor algorithm of rotor speed error. For 
example, if the rotor speed is not exceeding the maximum RPM but the rotor acceleration is large, the PD output could be large due to the acceleration component. Because the acceleration is so great, the PD is predicting that the rotor speed will soon exceed the maximum RPM setpoint, and the yaw motor starts turning at a high yaw rate in order to get the wind machine out of the wind quickly. Results of the three controllers are shown below in Figure 6.

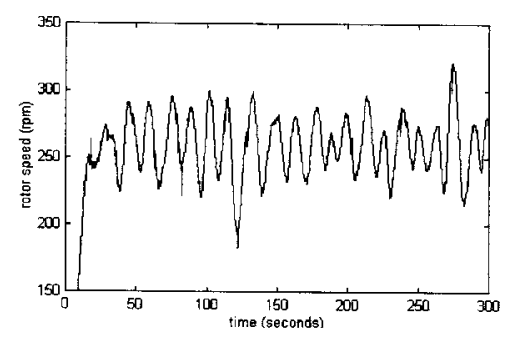

(a)

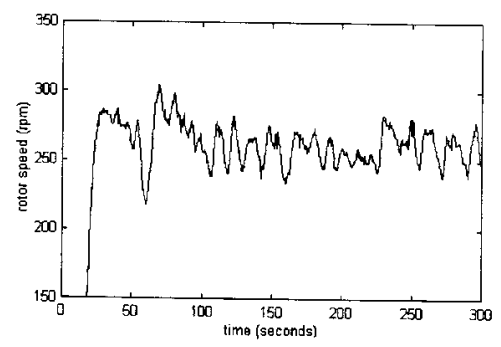

(b)

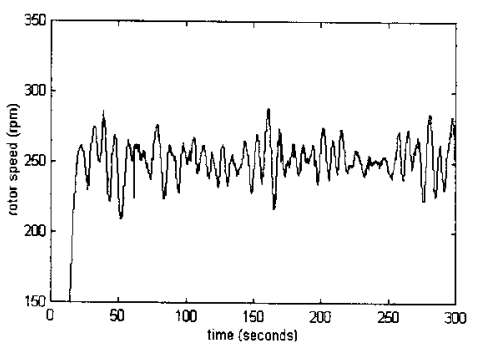

(c)

Figure 6 (a) First Controller, (b) Second Controller, (c) Third Controller

The yaw control system was also used as a design project in a senior digital systems class. Students were to input the wind, yaw, and rotor speed data into their digital controller and output the control signals to the yaw motor. Their controllers were to be constructed by using only logic gates, comparitors, counters, and timers. They had to convert the frequency signal from the anemometer into commands to start turning the wind machine into the wind (cut-in) or to turn the machine out of the wind in excessive winds (cut-out). The system also had to track the wind when the rotor was not in overspeed and to turn the machine in and out of the wind in overspeed to limit rotor speed near the maximum RPM. They could not use ADCs, DACs, or frequency-to-voltage chips to construct the controller. The students had to demonstrate their projects in the lab with simulated signals and then at the wind energy test bed. The design experience was greatly enhanced by actually implementing the controller on the wind machine and then by watching the response. Wind energy conversion systems are dynamic and quite impressive since they are housed on top of tall towers. Students felt they were controlling a "real-world" engineering system.

The wind machine has also been used for design projects in a linear control systems course. Students designed yaw controllers and simulated their responses using Simulink. Since different linear and nonlinear models have been developed, these models can be used to test controller designs. Wind data recorded at the test bed can be used as input into the simulations. Once tested on the computer, the controllers can be implemented on the wind machine with minimal programming by using Basic 52. Linear models used in simulations can then be checked in the field to see how controllers work outside their linear operating points. These are valuable exercises in real-world control problems 
which are sometimes difficult to demonstrate in the lab or on the computer. Also used at Montana Tech is a software package called Vissim. This package is similar to Simulink and uses the block diagram modeling technique. However, it also has a real time I/O option. Inexpensive hardware was purchased for the personal computer to interface Vissim to analog input signals and to output both digital and analog control signals. Students can simulate control strategies they design and then directly implement that controller at the wind energy site. The PID-SCR alternator controller can also be used to demonstrate typical hardware used in industry and how that hardware can be implemented for different applications. This hands-on control and instrumentation experience should help students make the transition from academic life into industry.

A graduate research project is presently testing a fuzzy logic controller that uses neural networks to learn the best fuzzy parameters needed for control. Another graduate student is using the test bed to acquire the rotor blade strain encountered at different yaw angles. These strains are then used to simulate the rotor blade root stresses using a finite element analysis software package called Algor. Strains are duplicated in the lab on the rotor blade to get an experimental measure of applied forces. A Montana Tech professor, in partial fulfillment of his Ph.D. degree, is also using the test bed to perform research in fatigue-life prediction of composites.

Both graduate and undergraduate students have been supported through the grant during the summer and the school year. A total of four graduate students and 16 undergraduates have been hired over the past four years. Most of the construction and instrumentation work was performed in the summer. Up to four undergraduates and two graduate students have been employed during a single summer to perform tasks that range from welding and machine design to instrumentation and programming. Because some of the instrumentation is experimental and uses student designed circuits, a large amount of time is spent trouble-shooting the problems encountered due to signal noise, poor design, and errors in construction. Time spent trouble-shooting is both frustrating and rewarding for students, but it gives them a realistic introduction into engineering design. Many typical problems of the control industry are also encountered and sometimes amplified at the test bed. In the future, the wind energy test bed will be used to rapidly fatigue test fiberglass rotor blade designs. However, it will also continue to be a vital part of the control systems curriculum at Montana Tech for design projects. The wind machines at the test bed can demonstrate fluid dynamics, aerodynamics, machine design, power transmission, energy conversion, instrumentation, control systems, signal processing, data acquisition, and telecommunications. Using some ingenuity, Tech faculty can demonstrate most basic general engineering theory on the wind machine. Moreover, students appear to appreciate a field trip to the wind site. 


\section{Bibliography}

1. Nise, N.S., Control Systems Engineering, Addison-Wesley, 1995.

2. Johnson, C., Process Control Instrumentation Technology, Prentice-Hall, 1993.

3. Hilker, G.B., Modeling and Simulation of a Yaw Controlled Horizontal-Axis Wind Turbine, Montana Tech, 1996.

4. Franklin, G.F., Powell, J.D., Workman, M.L., Digital Control of Dynamic Systems, Prentice-Hall, 1994.

\section{Biographical Information}

\section{DAVID WESTINE}

Associate Professor and Head of the Engineering Science Department at Montana Tech of The University of Montana and holds a Ph.D. degree in Engineering, an M.S. degree in Electrical Engineering, and a B.S. degree in Mechanical

Engineering from North Dakota State University. Dr. Westine teaches in the Engineering Science Control Systems option and is the Project Leader of the Wind Energy Test Site, DOE-EPSCoR grant.

\section{GREG HILKER}

Control Systems Engineer for Ball Aerospace, Boulder, CO. and holds an M.S. degree in Engineering Science from Montana Tech of The University of Montana, and a B.S. degree in Mechanical Engineering from the University of Idaho. Mr. Hilker's graduate study was supported in part by the DOE-EPSCoR grant.

\section{FRED JENKINS}

Mechanical Engineer for Advanced Silicon Materials, Inc., Butte, MT and holds a B.S. degree in Engineering Science Control Systems option from Montana Tech of The University of Montana. Mr. Jenkins was supported in part as an undergraduate by the DOE-EPSCoR grant. 